\title{
Baroreceptor Function Revealed by Acute Sinoaortic Denervation in Spontaneously Hypertensive Rats
}

\author{
Myo Thant, Yukio Yamori, and Kozo Okamoto
}

\begin{abstract}
Kymographical recordings of acute effects of sinoaortic denervation on blood pressure in spontaneously hypertensive rats and normotensive Wistar rats revealed that the former showed a significantly greater pressor response than the latter after the denervation even at the age of 30-60 days, at the age corresponding to the prehypertensive stage while there was no significant difference in the pressor response between the former and the latter at the adult age. These results provide an evidence that primary defect or dysfunction of baroreceptors is not involved in the pathogenesis of spontaneous hypertension, and are rather suggestive of primary activation of vasomotor center as one of the probable causes of this hypertension.
\end{abstract}

$\mathrm{T}^{\mathrm{n}}$

HE IDEA ${ }^{1}$ that dysfunction of baroreceptors may play a part in the development of primary hypertension (essential hypertension) has been proposed. There is, however, no clinical evidence to prove this idea, which also does not seem to be widely favoured because of the lack of knowledge in the field of experimental hypertension.

Окамото et al. succeeded in obtaining spontaneously hypertensive rats by repeating selective sib-breeding.,4 After many years of their work on the development of spontaneously hypertensive rats they could now proliferate up to $F_{19}$ generations, 5 and all the offspring of spontaneously hypertensive rats showed $100 \%$ occurrence of moderate or severe hypertension with cardiovascular lesions. ${ }^{6}$ It is interesting to notice that these offspring maintain primary hypertension already at the age of 60 days after birth. Hypertension develops in these rats even in the absence of severe organ changes, especially in the cardiovas- cular-renal system before the occurrence of spontaneous hypertension. 6 As for the pathogenesis of spontaneous hypertension elaborate studies by ОКАMOTO and his coworkers have proved various findings suggestive of the participation of neural ${ }^{7,8,9}$ and endocrine ${ }^{10,11}$ factors. Concrete evidences ${ }^{7}$ of sympathetic hyperactivity in these rats led us to study on the function of their baroreceptors.

This paper is going to clarify whether dysfunction of baroreceptor primarily contributes to the sympathetic hyperactivity, i.e., hypertension in these rats as in experimentally induced neurogenic hypertension following sinoaortic denervation, or primary activation of sympathetic vasomotor center is mainly responsible for the development of the spontaneous hypertension.

\section{Materials}

One hundred sixty male and female offspring of Spontaneously Hypertensive Rats (SHR),

(Received for Publication, February 20, 1969)

Department of Pathology, Faculty of Medicine, Kyoto University, Kyoto, Japan. (Director: Prof. Kozo Okamoto)

* The outline of this work was reported at the 17th General Assembly of the Japan Medical Congress, April 1967, Nagoya. This study was supported in part by USPHS grant HE10762, and in part by a Grant-in-aid for Scientific Research from the Ministry of Education, Japan and the Chiyoda Mutual Life's Subsidiary Fund for Social Welfare Works. 
supplied from among $F_{14}-F_{17}$ generation of Okamoto's lineage, and 412 male and female normotensive control rats (NR) supplied from Animal Center Laboratory, Kyoto University, both bred from the Wistar strain, were used throughout this experiment. The conditions under which these rats were kept were the same as reported in previous papers ${ }^{3,4}$ except that the feeding was converted into stock chow, CA-1 and CE-2 (Japan CLEA Co.). All these healthy rats were selected at the ages of 30-40, 40-50, 50-60 and 90-120 (adult) days after birth, respectively.

\section{Methods}

Neurogenic hypertension can be produced in rat by KRIEGER's method of sinoaortic denervation. ${ }^{2}$ But his article did not discuss the very important problem of quantitative (survival of animals) or qualitative (100\% disruption of sinoaortic nerves) results, and did not reveal the acute effect of sinoaortic denervation. The report did not mention anything about the severe respiratory difficulty and sudden death in an anesthetized rat due to carotid sinus area denervation! ${ }^{3}$, 14,15 The following simple reliable technique was adopted to overcome the above problems and the operation was established through the confirmation of complete sinoaortic nerve sections by a simple kymographic recording of femoral pressure during the sinoaortic denervation.

The healthy group of rats was selected three days prior to this operation, and prophylactic antibiotics were given to all these animals two or three days before operation. The operation procedures were performed only on healthy rats. The operation was done under Nikon Zoom stereo microscope on the unanesthetized rat which had received $0.5 \mathrm{mg}$ atropine intraperitoneally 3 minutes prior to this operation.

The rat was tied up with four strong strings by both upper and lower extremities. Then it was immobilized on the disecting board. A soft string was attached to the upper incissor teeth and pulled to stretch the neck to a suitable position for further disection. The hairy neck was cleaned with $70 \%$ alcohol for the purpose of sterilization. The neck and femoral part were shaved, and then the skin surface was resterilized again. The animal was ready for the cannulation of femoral artery for the direct measurement of femoral pressure. The heparinized cannula (No. 3 Hibiki polyethylene tubing) was inserted into the femoral artery during temporary occlusion of this artery by a small clipper. The cannula was

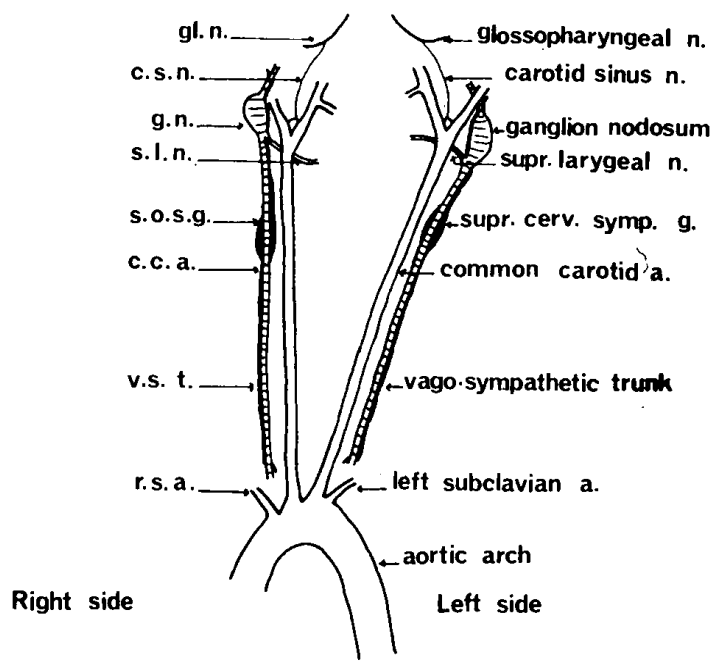

Fig.1. The Anatomical position and distribution of sinoaortic nerves in the rat.

tied up with this artery, and connected to the kymographic recording instrument and the initial blood pressure was checked up for 2 minutes.

The incision was performed at the center of neck and the subcutaneous connective tissue layer was exposed. The connective tissues were cut out straight between the left and right submaxillary glands, and the upper neck muscles (masseter and digastricus) and middle neck muscles (sternohyoideus and cleidomastoideus) were exposed. All the upper neck muscles were stretched out so that the upper layer of middle neck muscle layer was exposed. The middle neck muscle (sternohyoideus) was stretched to the left side, and then the right common carotid artery under the muscles of sternohyoideus and omohyoideus could be seen with its pulsatile movement. These two muscles were cut in the direction of the neck and the whole common carotid artery with its bifurcation was exposed. Along the lateral position of this artery, there could be seen a vago-sympathetic trunk as shown in Fig. 1. The fibrous connective tissues and neurovascular sheath were saparated out carefully. The selected sympathetic trunk was isolated from the vagus trunk and it was cut in the middle length for the aortic nerve denervation. The same procedure was performed on the other side again. Therefore, all the depressor nerves which ran along the sympathetic trunks were denervated. The right or left superior laryngeal nerve which originated from the base of ganglion nodosum ran beneath the carotid bifurcation and passed the lower border of this bifurcation could easily be identified. Both superior laryngeal nerves were 


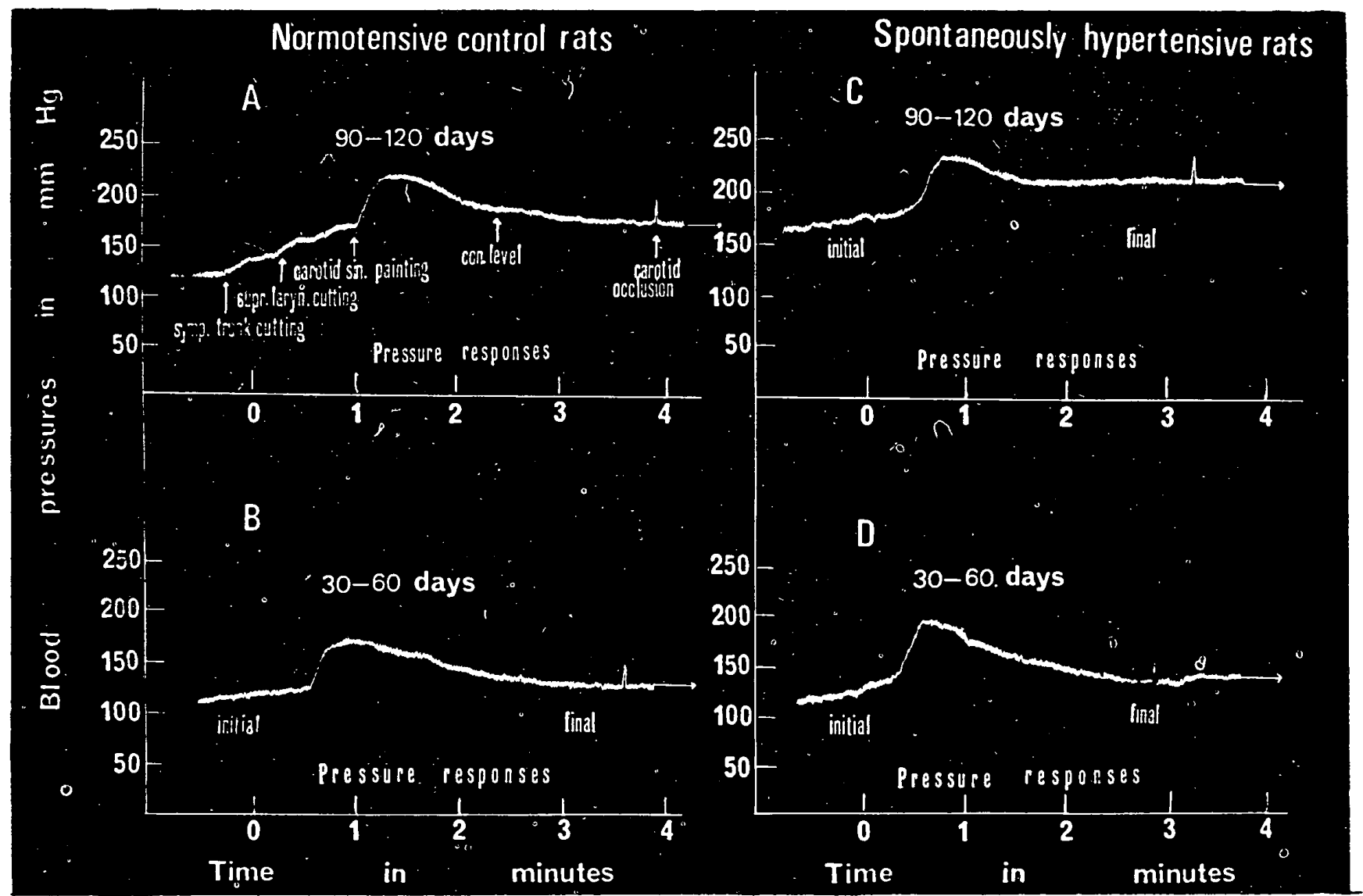

Fig. 2. Acute pressor responses following complete sinoaortic denervation in Spontaneously Hypertensive Rats and in normotensive control rats.

cut at midway, and the aortic depressor nerves which ran along these were denervated. The right bifurcation was cleaned out and stripped very carefully, and this bifurcation was painted with $10 \%$ phenolic alcohol solution. ${ }^{2}$ The left bifurcation was also treated in the same manner, and the total sinoaortic denervation was completed at last. After blood pressure was raised to a maximum level and returned to a constant level as recorded in the kymogram, the carotid occlusion tests for 20 seconds were performed just to check up on the nature of carotid sinus reflexes. The blood pressure tracing curves in the kymographic recording confirmed that buffer nerves were completely denervated.

The rat was disconnected from the kymographic recording instrument and the skin incisions were sutured. Bilateral sinoaortic denervation, cannulation of femoral artery and blood pressure recording were performed and the whole procedure lasted 45 minutes. After the operation each rat received approximately 10,000 to $12,000 \mathrm{IU}$ penicillin daily for 5-6 days for further study on blood pressures in the chronic stage. All these rats were fed on stock chow (CLEA, CA-1) and vegetables up to one month, and then were returned to ordinary diet (CLEA, CA-2).

Mean blood pressure of each unanesthetized rat was kymographically measured and all the data were examined statistically with Student's " $t$ " test, to confirm the significant differences.

\section{R ESULTS}

1) Sinoaortic denervation in normotensive control rats (Preliminary experiment)

Pressor responses during sinoaortic denervation done by the above-mentioned technique was kymographically observed in adult NR as shown in Fig. 2. The blood pressure increased stepwise following the serial disruption of bilateral sympathetic trunks and superior laryngeal nerves and abruptly rose to the peak after painting bilateral carotid sinus areas with $10 \%$ phenolic alcohol. Then, blood pressure returned to the constant level, which was higher than the initial level of blood pressure and was maintained for several hours during the kymographical observation. According to our preliminary observations on the acute and chronic effect of sinoaortic denervation in bulk of denervated rats, a group of rats 


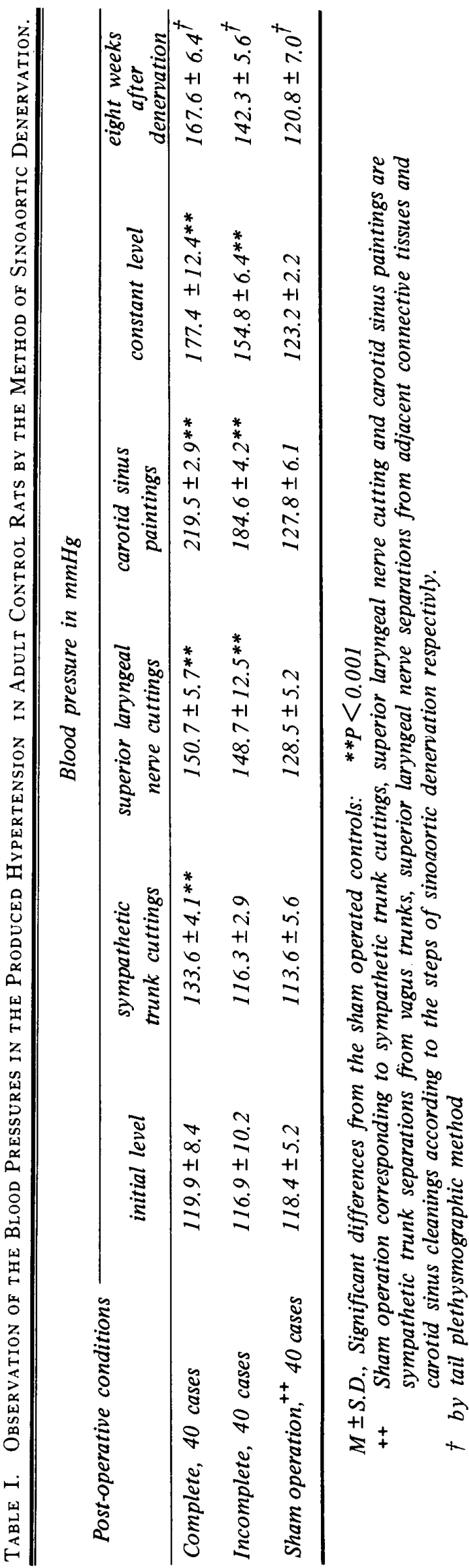

which showed pressor response following sympathetic trunk cutting was prone to reach higher constant level after painting the baroreceptor area and to develop chronic hypertension. This group of rats was tentatively designated as a completely and incompletely denervated groups are summarized in Table I. The removal of all buffer nerves in the acute experiment resulted in a rise of blood pressure from $119.9 \pm 8.4 \mathrm{mmHg}$ to $219.5 \pm 2.9 \mathrm{mmHg}$, and the pressure came down to a constant level of $177.4 \pm 12.4 \mathrm{mmHg}$ in all completely denervated control rats of 90-120 days old. The chances to obtain completely denervated rats were statistically less in these Wistar rats $(19.8 \%)$ because the route of aortic depressor nerves running through the sympathetic trunks might be few. In the incompletely denervated cases the blood pressure, after all procedures of denervation, stayed at the peak level of $184.6 \pm 4.2 \mathrm{mmHg}$, and then it gradually dropped to a constant level of $154.8 \pm 6.4$ $\mathrm{mmHg}$. These pressure levels were evidently lower than those in the completely denervated rats. In the following results the incompletely denervated group was neglected.

(a) Bilateral sy mpathetic trunk excisions in NR

About twenty per cent of experimental animals (Wistar rats) denervated by the above technique showed a definite slight elevation of blood pressures. They were regarded as being completely denervated, for aortic depressor nerves included in sympathetic trunks were confirmed to be cut by pressor response following sympathetic trunk cutting.

(b) Bilateral superior laryngeal nerve excisions in NR

One hundred per cent of the rats denervated by this technique showed very effective blood pressure responses and the blood pressures were remarkablely higher than the sham operated rats, the blood pressure of which were observed kymographically under the same operative conditions except for sinoaortic denervation.

(c) Chemical denervation (10 per cent phenolic alcohol painting) in NR

All rats showed extremely sensitive responses to this technique. After the maximum rise blood pressure stayed in the peak level for few minutes (at least 2 minutes) and fell to a constant level which continued for several hours as long as it was kymographically recorded. The difference between this constant level and the initial blood pressure was designated as constant rise in this article. 
Table II. Blood Pressure Responses to Sinoaortic Denervation in Spontaneously Hypertensive Rats at the Pre- and Early Hypertensive Stages and the Age-matched Control Rats.

\begin{tabular}{llllll}
\hline \multirow{2}{*}{ Animals (No.) } & Ages in days & \multicolumn{4}{c}{ Blood pressure in $\mathrm{mmHg}$} \\
\cline { 3 - 6 } & & initial level & maximum level & constant level & constant rise \\
\hline spontaneously & $30-40$ & $118.4 \pm 5.2$ & $161.1 \pm 10.4^{* *}$ & $150.8 \pm 4.4^{* *}$ & $32.4 \pm 6.0^{* *}$ \\
hypertensive & $40-50$ & $122.6 \pm 12.2$ & $156.0 \pm 2.9^{* *}$ & $151.1 \pm 2.9^{* *}$ & $28.5 \pm 5.6^{*}$ \\
rats (40 cases) & $50-60$ & $129.8 \pm 7.7$ & $190.5 \pm 9.4^{* *}$ & $166.3 \pm 10.6^{* *}$ & $36.5 \pm 6.4^{*}$ \\
& $90-120$ & $157.6 \pm 6.2^{* *}$ & $214.1 \pm 5.1$ & $207.8 \pm 6.1^{* *}$ & $50.2 \pm 7.5$ \\
\hline normotensive & $30-40$ & $121.6 \pm 5.2$ & $147.5 \pm 5.2$ & $132.4 \pm 2.4$ & $10.8 \pm 1.2$ \\
control & $40-50$ & $121.6 \pm 5.7$ & $149.8 \pm 1.2$ & $134.1 \pm 4.0$ & $12.5 \pm 3.7$ \\
rats (40 cases) ${ }^{+}$ & $50-60$ & $123.2 \pm 2.9$ & $152.3 \pm 2.7$ & $143.6 \pm 5.3$ & $20.4 \pm 4.8$ \\
& $90-120$ & $123.6 \pm 3.2$ & $212.0 \pm 8.1$ & $173.0 \pm 3.0$ & $49.4 \pm 7.4$
\end{tabular}

\footnotetext{
$M \pm S . D .$, Significant differences from the controls: $\quad * * P<0.001, \quad * 0.001<P<0.01$ 10 rats in each group
}

2. Sinoaortic denervation in spontaneously hypertensive rats compared with that in normotensive control rats.

SHR and NR were used to investigate the features of baroreceptor activity especially at the prehypertensive and early hypertensive stage of SHR and at the corresponding age of NR. The results in Table II are for the comparison of SHR with the age-matched NR. The SHR which showed a pressor response after sympathetic trunk cutting were regarded as being completely denervated as mentioned above in NR. The results of Table II were selected from the completely denervated SHR while the incompletely denervated SHR were neglected from this experiment.

The initial blood pressures in young SHR from 30 to 50 days after birth were not significantly different and not so greatly different even at the age of 50 to 60 days from those in the agematched NR. However, the responses after sinoaotric denervation, i.e., maximum and constant level, and constant rise were all significantly greater in these young SHR than in young NR.

On the other hand, adult SHR after the development of spontaneous hypertension had significantly higher initial level than the adult NR, and the constant level after the denervation was also significantly higher in the former than in the latter. But the pressor responses after the deprivation of baroreceptor function as shown in constant rise were almost the same both in the former and the latter.

\section{DISCUSSION}

The main difficulty in complete sinoaortic denervation in rats is due to irregurality of the course of aortic depressor fibers. ${ }^{16}$ Aortic depressor fibers of the rat usually run along the right and left vago-sympathetic trunks.17,18 By cutting the right and left sympathetic trunks and both superior laryngeal nerves, almost all of the fibers of aortic baroreceptor can be denervated and a considerable rise of blood pressure is detected from the femoral artery by a kymographic recording instrument. The carotid baroreceptor areas can be denervated by painting the areas of both carotid bifurcations with phenolic alcohol solution. The sign of complete removal of sinoaortic nerves in the rat can be detected by the kymographic recording, which shows continuous rise of blood pressure up to $219.5 \pm 2.9 \mathrm{mmHg}$ and a constant blood pressure level of 177.4 $\pm 12.4 \mathrm{mmHg}$ in $90-120$ day old control rat in this experiment. However, when the aortic denervation is incomplete even after cutting both sympathetic trunks and both superior laryngeal nerves, aortic depressor fibers may be present in the vagus trunks ${ }^{17}$ or indifferent routes instead of sympathetic trunks. Here, in this experiment, 19.8 per cent of these Wistar rats only showed pressure responses after sympathetic trunk cutting. In view of these findings it is considered to be worthwhile to determine the course of blood pressure changes which occur after the denervation of aortic and carotid sinus baroreceptors. Kymographic recording technique is rather reliable to confirm the complete denervation.

In the course of this experimental denervation of carotid sinus regions, almost all of the rats died of respiratory failure when they were treated with any kind of anesthetics in the 
dosages sufficiently enough or less enough to produce surgical anesthesia, because all kinds of general anesthetics can depress respiration. ${ }^{15,19}$ These observations explain the sudden death encountered in anesthetized rats during or after denervation of carotid sinus regions, and the operation without anesthesia could avoid the sudden death. Our observations also showed that the denervation of sinoaortic baroreceptors was not fraught with danger at least in healthy and unanesthetized rats. There was neither respiratory death in these completely denervated rats nor a single instance of "cardiac" death suggested byHERING ${ }^{20}$ This finding supported the results obtained by WITT et al! ${ }^{13}$

Consequently, in order to obtain the quantitative (survival of animals) or qualitative (completely denervated animals) results, two conditions have to be adopted: first, the rat must not be treated with any kind of general anesthetics; second, the real disruption of aortic fibers in both sympathetic trunks must be confirmed by checking blood pressure during denervation.

As shown in Table II, it is especially interesting to observe the results obtained by denervation in control rats and SHR. Only the data of completely denervated controls and SHR are included in this result so that the quantitative comparison of the effect of denervation between controls and SHR is possible. Firstly, the pressor responses after sinoaortic denervation were significantly greater in young SHR at 30-60 days than in the age-matched NR, while their initial blood pressures were not different from each other. Secondly, adult SHR which had significantly higher initial blood pressure than adult NR, showed almost the same pressor response after the denervation as that in the NR group.

The first evidence is clearly incompatible with the idea that primary defect or dysfunction of baroreceptor is involved in the cause of the sympathetic hyperfunction or hypertension in SHR. This fact rather favours a probable assumption of primary activation of sympathetic system or vasomotor center in SHR, if other factors such as vascular reactivity to endogenous neurohumor are not concerned in SHR. Such hyperfunction of vasomotor center could be disclosed only by the deprivation of buffer function which may dynamically counteracts against the activated function of vasomotor center before the development of hypertension. It could be speculated that hypertension would develop when such dynamic balance is lost and transformed into a new balance proved by the second evidence. The second evidence supported that baroreceptor in SHR is functioning roughly with normal capacity even after hypertension has been established. Baroreceptor function in SHR could be regarded as being reset at a higher level of blood pressure. It is now clear at least that SHR is completely different from experimental neurogenic hypertension obtained by sinoaortic denervation which develops owing to sympathetic hyperfunction in the absence of buffer function.

The present experiments elucidated a little the baroreceptor function in SHR and indicated the possible dynamic balance between vasomotor center and buffer function before, during and after the development of hypertension. Further detailed analysis of baroreceptor function in SHR $^{21}$ is of great interest also for understanding the role of baroreceptor in essential hypertension.

\section{S UMMARY}

The function of baroreceptors in 160 offspring of spontaneously hypertensive rats and 412 normotensive Wistar rats (control rats) was investigated by the method of sinoaortic denervation without anesthesia. The direct blood pressure was recorded kymographically through the femoral artery during this operation. The following results were obtained after the complete sinoaortic denervation:

1) Acute hypertension ( $177.4 \pm 12.4$ or $173.0 \pm$ $3.0 \mathrm{mmHg}$ ) was produced in $90-120$ day old control rats by the method of sinoaortic denervation performed under the kymographic observation on acute changes in blood pressure while the younger control rats, 30-60 day old, showed less pressor response (constant rise, $10-20 \mathrm{mmHg}$ ) and failed to develop acute hypertension $(132-143 \mathrm{mmHg}$ in average) by using the same procedure.

2) Spontaneously hypertensive rats at the age of 30-60 days showed a greater pressor response (constant rise, $32-36 \mathrm{mmHg}$ ) and maintained a higher constant level $(150-166 \mathrm{mmHg}$ in average) after the denervation than in the agematched normotensive Wistar rats. Adult spontaneously hypertensive rats, however, showed almost the same pressor response $(50.2 \pm 7.5$ $\mathrm{mmHg})$ as the normotensive rats $(49.4 \pm 7.4$ $\mathrm{mmHg}$ ), while the constant level after the denervation was evidently higher in the former (207.8 $\pm 6.1 \mathrm{mmHg})$ than in the latter $(173.1 \pm 3.0$ $\mathrm{mmHg}$ ).

3) These comparisons of acute effects of the 
sinoaortic denervation in spontaneously hypertensive rats and in normotensive rats supported an idea that primary defect or dysfunction of baroreceptor function was not responsible for the development of spontaneous hypertension, and favoured an assumption of probale primary activation of vasomotor center. The baroreceptor in adult spontaneously hypertensive rats was confirmed to be functioning roughly with normal capacity at the high level of blood pressure.

4) A reliable method for sinoaortic denervation was established by the following devices.

a) The complete disruption of sinoaortic nerves was confirmed by a simple kymographic recording.

b) Mortality rate during and after the denervation was greatly diminished by operating on rats without anesthesia and by prophylactic administration of antibiotics.

\section{REFERENCES}

1. Horrobin D. F.: A theory of hypertension. Lancet I: $574-579,1966$.

2. Pickering, G. W.: Hyperpiesis: high blood-pressure without evident cause: essential hypertension, Brit. Med.J. 2 : 959-968, 1965.

3. Oкамото, K. \& Aoki, K.: Development of a strain of spontaneously hypertensive rats. Japan. Circul. J. 27 : 282-293, 1963.

4. OKamoto, K., Tabei, R., Fukushima, M., Nosaka, S., Yamori, Y., Ichijima, K., HaEbara, H., Matsumoto, M., Maruyama, T., Suzuki, Y., \& TAMEgar, M.: Further observations on the development of a strain of spontaneously hypertensive rats. Japan. Circul. J. 30: 703-716, 1966.

5. Окамото, K.: Spontaneous hypertension in rats. International review of experimetal pathology, 7 : 227-268, Academic Press Inc., New York and London, 1969.

6. Окамото, K., Аoki, K., Nosaka, S., \& Fukushima, M. : Cardiovascular diseases in the spontaneously hypertensive rat. Japan. Circul. J. 28: 943-952, 1964.

7. OKamoto, K., Nosaka, S., Yamori, Y., \& Matsuмото, M.: Participation of neural factor in the pathogenesis of hypertension in the spontaneously hypertensive rat. Japan. Heart J. 8 : 168-180, 1967.
8. OKamoto, K., Hazama, F., Takeda, T., Tabei, R., Nosaka, S., Fukushima, M., Yamori, Y., Matsumoto, M., Haebara, H., Ichijima, K., \& Suzuki, Y.: Pharmacodynamic studies on the cardiovascular system of spontaneously hypertensive rats. Japan. Circul. J. 30 : 987-1007, 1966.

9. Matsumoto, M.: Morphological studies on the autonomic nervous system of hypertensive rats. I. Histochemical study on the superior cervical sympathetic ganglion of spontaneously hypertensive rats. Japan. Circul. J. 30: 743-753, 1966.

10. Aoki, K., Tankawa, H., Fujinami, T., Miyazaki, A., \& Hashimoto, Y.: Pathological studies on the endocrine organs of the spontaneously hypertensive rats. Japan. Heart J. 4 : 426-442, 1963.

11. Aok , K.: Experimental studies on the relationship between endocrine organs and hypertension in spontaneously hypertensive rats. I. Effects of hypophysectomy, adrenalectomy, thyroidectomy, nephrectomy and sympathectomy on blood pressure. Japan. Heart J. $4:$ 443-461, 1963.

12. KRIEGER, E. M.: Neurogenic hypertension in the rat. Circul. Res. 15: 511-521, 1964.

13. Witt, D. B., Katz, L.N., \& KoHn, L.: Respiratory failure following denervation of the carotid sinus regions. Amer. J. Physiol. 107 : 213-219, 1934.

14. Gemmili, C. L., Overstreet, E. W., \& Hellman, L.M.: The effect of occlusion of the carotid arteries on heart rate and respiratory rate before and after denervation of the carotid sinus in normal dogs. Amer. J. Physiol. 104 : 443-448, 1933.

15. WANG, S. C., \& NimS, L. F.: The effect of various anesthetics and decerebration on the $\mathrm{CO}_{2}$ stimulating action on respiration in cats. J. Pharmacol. Expt. Therap. 92: 187-195, 1948.

16. ANDREw, B. L.: A laryngeal pathway for aortic baroreceptor impulses. J. Physiol. (London) 125 : 352-360, 1954.

17. McCubin, J. W., Masson, G. M.C., \& Page, I.H. : Aortic depressor nerves of the rat. Arch. Inter. Pharmacodyn. 114: 303-306, 1958.

18. Krieger, E. M. \& Marseillan, R. F.: Aortic depressor fibers in the rat: an electrophysiological study. Amer. J. Physiol. 205: 771-774, 1963.

19. Cozine, R. A. \& NGaI, S. H.: Medullary surface chemoreceptors and regulation of respiration in the cat. J. Appl. Physiol. 22 : 117-121, 1967.

20. Hering, H. E. : Die Karotissinusreflexe auf Herz und Gefässe. Verlag von Theodor Steinkopff, Dresden und Leipzig, 1927.

21. Nosaka, S. \& Okamoto, K. : Modified characteristics of the baroreceptor activities of the spontaneously hypertensive rat. (in preparation). 